\title{
UPPER BOUNDS ON HOMOLOGICAL DIMENSIONS
}

\section{B.L. OSOFSKY}

The homological dimension of a module $M_{R}$ is often related to the cardinality of a set of generators for $M$ or for right ideals of $R$. In this note, upper bounds for this homological dimension are obtained in two situations.

In [8] Jensen has shown that, for any ring $R$ whose finitely generated right ideals are countably related, if any right ideal of $R$ is generated by $\boldsymbol{\aleph}_{n}$ elements, then the right global dimension of $R$ exceeds the weak global dimension by at most $n+1$. In section 1 we show that the condition that finitely generated right ideals are countably related may be deleted, and Jensen's theorem will still hold.

In [3] Berstein showed that a direct limit of modules over a countable directed system has dimension at most one more than the supremum of the dimensions of the modules. This is also an immediate consequence of Roos [14], Theorem 1. In section 2 we show that a direct limit of modules over a directed system of cardinality $\boldsymbol{\aleph}_{n}$ has dimension at most $n+1$ more than the supremum of the dimensions of the modules. Balcerzyk showed this for a directed union in [2].

All rings $R$ will have identity 1 ; all modules will be unital right $R$ modules. For a module $M, h d_{R}(M)$ (or $h d(M)$ if no confusion arises) will denote the homological dimension of $M$. $g l . \mathrm{d}(R)$ will denote the right global dimension of $R$ and w. gl. $\mathrm{d}(R)$ its weak global dimension. A basic tool for calculating upper bounds on homological dimensions is the following proposition of Auslander.

Proposition 0.1. Let $\mathscr{I}$ be a non-empty well-ordered set, $M$ a right $R$ module, $\left\{N_{i} \mid i \in \mathscr{I}\right\}$ a family of submodules of $M$ such that $N_{i} \subseteq N_{j}$ for $i \leq j$. If $M=\cup_{i \in \mathscr{I} N_{i}}$ and $h d_{R}\left(N_{i} / \cup_{j<i} N_{j}\right) \leq n$ for all $i \in \mathscr{I}$, then $h d_{R}(M) \leq n$.

Proof. This is proposition 3 of [1].

Received September 18, 1967.

This paper was written while the author held a National Science Foundation Postdoctoral Fellowship and a Rutgers University Research Council Faculty Fellowship. 
Proposition 0.2. Let $B_{R} \subseteq A_{R}$. If $h d(A)>h d(B)$, then $h d(A)$ $=h d(A / B)$. If $h d(A)<h d(B)$, then $h d(A / B)=h d(B)+1 . \quad$ If $h d(A)=h d(B)$, then $h d(A / B) \leq 1+h d(A)$.

Proof. This is Theorem 1. 2 of Kaplansky [10].

We observe that $h d(B)$ cannot exceed both $h d(A)$, and $h d(A / B)$, and $h d(B)=h d(A / B)$ implies $h d(B)=h d(A)$.

Corollary 0.3. Under the hypotheses of Proposition 0.1, if $h d_{R}\left(\cup_{j<i} N_{j}\right)$ $\leq m$ for all $i \in \mathscr{I}$, then $h d_{R}(M) \leq m+1$.

Proof. Since $h d_{R}\left(\cup_{j<i} N_{j}\right) \leq m$ and $h d_{R}\left(N_{i}\right)=h d_{R}\left(\cup_{j<i+1} N_{j}\right) \leq m, h d_{R}\left(N_{i}\right)$ $\left.\cup_{j<i} N_{j}\right) \leq m+1$ by Proposition 0.2 . Hence $h d_{R}(M) \leq m+1$ by Proposition 0.1 .

§ 1. Dimension of a flat module. In this section we get an upper bound on the homological dimension of a flat $R$-module in terms of the number of generators of ideals of $R$.

Definition : $R$ is called $\aleph_{n}$-noetherian if every right ideal of $R$ is generated by a set of elements of cardinality $\leq \boldsymbol{\aleph}_{n}$.

Lemma 1. 1. Let $F$ be an $R$-module generated by a set of cardinality $\leq \aleph_{n}$. If $R$ is $\boldsymbol{\aleph}_{n}$-noetherian, then every submodule of $F$ is $\boldsymbol{\aleph}_{n}$-generated.

Proof. Jensen's proof in [7] for the case $n=0$ goes through exactly in this case.

Let $F$ be a free $R$-module. We say $B \subseteq F$ is a $*$-submodule of $F \Leftrightarrow$ for all $\left\{b_{i} \mid 1 \leq i \leq n\right\} \subseteq B$, there is a $u \in \operatorname{Hom}_{R}(F, B)$ such that $u\left(b_{i}\right)=b_{i}$ for $1 \leq i \leq n$. By [4], p. 65, $B$ is a $*$-submodule of $F \Leftrightarrow F / B$ is flat.

Lemma 1.2. Let $M$ be a countably related flat $R$-module. Then $h d_{R}(M)$ $\leq 1$.

Proof. See Jensen [7], Lemma 2.

Theorem 1. 3. Let $F$ be a free $R$-module with basis $\left\{x_{i} \mid i \in \mathscr{I}\right\}, B a *-$ submodule of $F$. Then any submodule $T$ of $B$ generated by $\boldsymbol{\aleph}_{k}$ elements can be

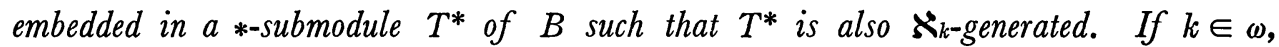

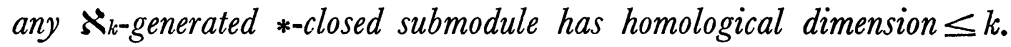


Proof. We use transfinite induction on $k$.

Basis : $k=0$.

Let $\left\{t_{i} \mid i \in \omega\right\}$ generate $T \subseteq B$. We inductively define $T_{i}, u_{i}$ so that $T_{0}=t_{0} R$ and

i) $t_{i} \in T_{i}$

ii) $T_{j} \subseteq T_{i}$ for all $j \leq i$

iii) $\quad T_{i}$ is a finitely generated submodule of $B$

iv) $u_{i}: F \longrightarrow T_{i+1}$ is the identity on $T_{i}$.

Assume we have $T_{i}$. Let $u^{\prime}: F \longrightarrow B$ be any map which is the identity on $T_{i}+t_{i+1} R$. Define $u_{i}: F \longrightarrow B$ by $u_{i}\left(x_{j}\right)=0$ if $T_{i}+t_{i+1} R$ has zero projection on $x_{j} R ; u_{i}\left(x_{j}\right)=u^{\prime}\left(x_{j}\right)$ otherwise. Since $T_{i}+t_{i+1} R$ is finitely generated, $u_{i}\left(x_{j}\right)=0$ for all but a finite number of $j \in \mathscr{J}$. Hence $T_{i+1}=u_{i}(F)$ is finitely generated and $\left\{T_{j} \mid 0 \leq j \leq i+1\right\}$ satisfies $\left.\mathrm{i}\right) \longrightarrow \mathrm{iv}$ ).

Set

$$
T^{*}=\cup_{i=0}^{\infty} T_{i}
$$

Since any finite subset of $T^{*}$ is contained in some $T_{i}, u_{i}: F \longrightarrow T_{i+1} \subseteq T^{*}$ is a map leaving it fixed. Hence $T^{*}$ is a $*$-submodule of $B$. Clearly $T^{*}$ is countably generated and contains $T$. Since $F / T^{*}$ is flat, by Lemma 1. $2, T^{*}$ must be projective.

Induction step. Let $\left\{t_{r} \mid \gamma<\aleph_{k}\right\}$ generate $T$. Set

$$
T_{\gamma}=\left[t_{\gamma} R+U_{\beta<\gamma} T_{\beta}\right]^{*}
$$

for all $\gamma<\aleph_{k}$. If for all $\beta<\gamma, T_{\beta}$ is generated by at most $\boldsymbol{\aleph}_{0}$ times cardinality of $\beta$ elements, then $t_{\tau} R+\cup_{\beta<\gamma} T_{\beta}$ is generated by $\boldsymbol{\aleph}_{0} \cdot$ cardinality $\gamma$ elements. Hence by the induction hypothesis, $T_{\gamma}$ exists and is generated by at most $\aleph_{0} \cdot$ cardinality $\gamma$ elements. Set

$$
T^{*}=U_{\gamma<\aleph_{k}} T_{\gamma}
$$

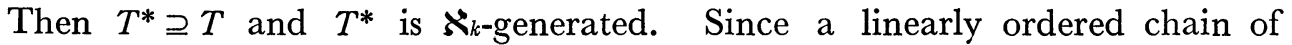
*-submodules is a $*$-submodule, $T^{*}$ is the required module.

If $k \in \omega$, since $\cup_{\beta<\gamma} T_{\beta}$ is a $*$-submodule of $F$ generated by a set of $\aleph_{k-1}$ elements, by the induction hypothesis $h d_{R}\left(\cup_{\beta<\gamma} T_{\beta}\right) \leq k-1$. By Corollary $0.2, h d_{R}\left(T^{*}\right) \leq k$. 
Corollary 1. 4. Let $R$ be $\aleph_{n}$-noetherian. Then

$$
g l . d(R) \leq w . g l . \operatorname{dim}(R)+n+1 .
$$

Proof. Let $I$ be any right ideal of $R$. Since $R$ is $\boldsymbol{\aleph}_{n}$-noetherian, we can find a projective resolution

$$
\cdots \longrightarrow F_{n} \longrightarrow \cdots \longrightarrow F_{0} \longrightarrow R / I \longrightarrow 0
$$

of $R / I$ where each $F_{n}$ is free on $\aleph_{n}$ generators. Let w. gl. $\operatorname{dim}(R)=k$. Then the image of $F_{k}$ is flat, so the image of $F_{k+1}$ is a $*$-submodule of $F_{k}$ generated by at most $\aleph_{n}$ elements. Then $h d_{R}\left(\right.$ Image $\left.F_{k+1}\right) \leq n$ by the theorem, so $h d_{R}(R / I) \leq k+1+n$. The global dimension theorem (Auslander [1]) completes the proof.

A module $M$ is flat if and only if $M$ is a direct limit of finitely generated free modules. (See [11].) Of course, direct limit cannot be replaced by directed union here. However:

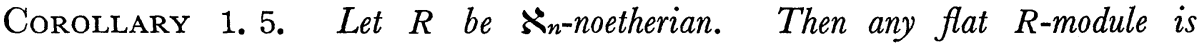
the directed union of $\boldsymbol{\aleph}_{n}$-generated flat submodules.

Proof. Let $N$ be any $\boldsymbol{\aleph}_{n}$-generated submodule of the flat module $M$, and let $F=\Sigma_{i \in \mathscr{F}} \oplus x_{i} R$ be a free module mapping onto $M$ with kernel $K$. We wish to find a flat submodule $N^{\prime} \subseteq M$ such that $N \subseteq N^{\prime}$ and $N^{\prime}$ is generated by $\aleph_{n}$ elements. We apply a snaking argument of Kaplansky [9]. $N$ is contained in the image of $\boldsymbol{\aleph}_{n}$ of the $x_{i}$, say $\sum_{i \in \mathscr{F}_{0}} x_{i} R$. Let $T_{0}=K \cap \Sigma_{i \in \mathscr{I}_{0}} x_{i} R . \quad T_{0}$ is $\boldsymbol{\aleph}_{n}$-generated by Lemma 1.1 . Hence $T_{0}$ is contained in an $\boldsymbol{\aleph}_{n}$-generated $*$-closed submodule $T_{1}$.

Assume $T_{l}$ has been defined so that $T_{l}$ is an $\aleph_{n}$-generated, $*$-closed submodule of $K$ containing $T_{j}$ for all $j<l$. Since $T_{l}$ is $\aleph_{n}$-generated, it is contained in $\sum_{i \in \mathscr{I}_{l}} x_{i} R$ for some set $\mathscr{I}_{l} \subseteq \mathscr{F}$ of cardinality at most

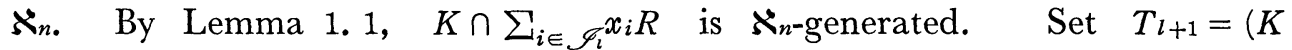
$\left.\cap \sum_{i \in \mathscr{I}_{i}} x_{i} R\right)^{*}$. Set

$$
\begin{gathered}
T=\cup_{l=0}^{\infty} T_{l} \\
\mathscr{I}^{\prime}=\bigcup_{l=0}^{\infty} \mathscr{I}_{l} .
\end{gathered}
$$

Then $T$ is an ascending union of *-closed submodules of $F$ and so *-closed. 
Moreover, $x \in K \cap \Sigma_{i \in \mathscr{I}}, x_{i} R$ implies $x \in \Sigma_{i \in \mathscr{I}_{i}} x_{i} \cap K$ for some $l$, so $x \in T_{l+1}$. Hence

$$
T=K \cap \Sigma_{i \in \mathscr{J}}, x_{i} R
$$

and $\sum_{i \in \mathscr{F}}, x_{i} R / T$ is isomorphic to a flat, $\aleph_{n}$-generated submodule of $M$ containing $N$. $M$ is the directed union of such submodules.

We note that Corollary 1.4 is the best possible result, for by Osofsky [12], an $\aleph_{n}$-noetherian valuation ring which is not $\aleph_{n-1}$-noetherian has global dimension $n+2$. Its weak dimension is 1 . By Pierce [13] a free boolean ring on $\aleph_{n}$ generators has global dimension $n+1$, and its weak dimension is 0 . It is unknown if the number of generators of the flat submodules can be reduced in 1.5. We can show, however, that in the case $n=0$, Corollary 1.5 is best possible. The following modification of an example of Kaplansky is due to C.U. Jensen.

Let $R$ be the subring of the ring of all continuous functions from $\boldsymbol{Q}$ to $\boldsymbol{Q}$ generated by functions composed of a finite number of linear pieces. Then $R$ is countable, and for all $r \in R, r^{-1}(0)$ consists of a finite number of components. Let $I$ be the ideal of $R$ consisting of functions vanishing in a neighborhood of 0 . Let $f_{0}=0$, and for $n \geq 1$, let $f_{n}$ be 1 on $(-\infty,-2 / n] \cup[2 / n, \infty)$, linear on $[-2 / n,-1 / n] \cup[1 / n, 2 / n]$, and 0 on $[-1 / n, 1 / n]$. Then $\left\{f_{n+1}-f_{n} \mid n \in \omega\right\}$ forms a dual basis for $I$, so $I$ is projective. We show that no finitely generated submodule of $I$ is flat.

Let $g_{1}, \cdots, g_{n} \in I$, and let $[a, b]$ be the largest neighborhood of 0 on which all of the $g_{i}$ vanish. By multiplying by -1 if necessary, we may assume all of the $g_{i}$ are non-negative on a neighborhood to the right of $b$, and at least one of the $g_{i}$ is strictly positive there. Let $h$ be non-zero on $(a, b)$ and zero elsewhere. Then $h g_{i}=0$ for all $i$. If $\sum g_{i} R$ is flat, the kernel of an epimorphism from a free module to it is a $*$-submodule, so there exist $\left\{p_{i j} \mid 1 \leq i, j \leq n\right\} \subseteq R$ such that $0=\sum_{i=1}^{n} g_{i} p_{i j}$, and $h p_{i j}=h \delta_{i j}$ for all $j, 1 \leq j \leq n$. Then

$$
0=\sum_{i=1}^{n} g_{i}\left(\sum_{j=1}^{n} p_{i j}\right)
$$

and $p_{i j}=\delta_{i j}$ on $[a, b]$. Hence $\sum_{j=1}^{n} p_{i j}=1$ on $[a, b]$, so in a neighborhood to the right of $b$, each $g_{i}\left(\sum p_{i j}\right) \geq 0$, and some $g_{i}\left(\sum p_{i j}\right)>0$, a contradiction. 
Corollary 1.6. Let $R$ have cardinality $\boldsymbol{\aleph}_{n}$. Then the left and right global dimensions of $R$ can differ by at most $n+1$.

Proof. Each dimension is equal to or greater than the weak dimension, and exceeds it by at most $n+1$.

In Corollary 1. 6, no smaller bound is possible, for Jategaonkar [6] has an example of a left hereditary ring of cardinality $\aleph_{n}$ and right global dimension $=n+2$.

§ 2. Dimension of a direct limit. Let $D$ be a directed set, $\left\{R_{i}, \pi_{i}^{j} \mid i, j \in D\right\}$ a directed system of rings, $\left\{M_{i}, \xi_{i}^{j} \mid i, j \in D\right\}$ a directed system of groups such that each $M_{i}$ is an $R_{i}$-module and $\xi_{i}^{j}(m r)=\xi_{i}^{j}(m) \pi_{i}^{j}(r)$ for all $m \in M_{i}, \quad r \in R_{i}$. Let $M=\underline{\lim } M_{i}, R=\underline{\lim } R_{i}$.

Proposition 2.1. If $D$ is countable, then $h d_{R}(M) \leq 1+\sup h d_{R_{i}}\left(M_{i}\right)$ and gl. $d(R) \leq 1+$ supgl. $d\left(R_{i}\right)$.

Proof. See Berstein [3].

We generalize this result to any cardinal $\aleph_{n}$, where $n \in \omega$.

Let $D^{\prime}=\left\{d_{r} \mid \gamma<\boldsymbol{\aleph}_{n}\right\}$ be cofinal in $D$. Then the direct limit over $D$ is the same as over $D^{\prime}$, so we may assume $D^{\prime}=D$.

Lemma 2. 2. Assume $n>0$. Then there exist directed subsets $\left\{E_{r} \mid \gamma\right.$ $\left.<\aleph_{n}\right\}$ of $D$ such that

$$
\begin{aligned}
& D=\cup_{r<\boldsymbol{\aleph}_{n}} E_{\gamma} \\
& E_{\beta} \subseteq E_{\gamma} \text { for } \beta<\gamma \\
& \text { Cardinality } E_{r}=\boldsymbol{\aleph}_{n-1} \text { for all } \gamma<\boldsymbol{\aleph}_{n} .
\end{aligned}
$$

Proof. Assume $E_{\beta}$ has been defined for all $\beta<\gamma$. Let $E_{\gamma, 0}=\left\{d_{\alpha} \mid \alpha\right.$ $\left.<\aleph_{n-1}+\gamma\right\} \cup \cup \beta<_{\gamma} E_{\beta}$. Then $E_{\gamma, 0}$ has cardinality $\aleph_{n-1}$. Once $E_{\gamma, i}$ has been defined, define $E_{r, i+1}=E_{\gamma, i} \cup F_{i}$, where $F_{i}$ is the image of a function from $E_{\gamma, i} \times E_{\gamma, i} \longrightarrow D$ taking $(x, y)$ to an upper bound of $x$ and $y$. Then each $E_{r, i}$ has cardinality $\aleph_{n-1}$, and so does $E_{r}=\cup_{i=0}^{\infty} E_{r, i}$. Clearly $D=\cup_{\gamma<\boldsymbol{\aleph}_{n}} E_{\gamma}$ and each $E_{\gamma}$ is a directed subset of $D$.

THEOREM 2. 3. If a set of cardinality $\boldsymbol{\aleph}_{n}$ is cofinal in $D$, then

$$
h d_{R}(M) \leq n+1+\operatorname{suph} h d_{R_{i}}\left(M_{i}\right)
$$




$$
g l . d(R) \leq n+1+\sup g l . d\left(R_{i}\right) .
$$

Proof. Berstein in [3] reduces the situation to the case where each $R_{i}=R$ and $\pi_{i}^{j}=$ the identity by first using induction to get each $M_{i}$ $R_{i}$-projective and then using the fact that $M_{R}$ is the direct limit of $\left\{M_{i} \otimes_{R_{i}} R\right\}$. So we may assume all $M_{i}$ are $R$-modules. We now use induction on $n$. The basis is Proposition 2. 1. Now assume $n>0$.

By Lemma 2. 2, $D=\cup_{\gamma<\aleph_{n}} E_{\gamma}$, where each $E_{\gamma}$ is a directed set of cardinality $\aleph_{n-1}$. Now

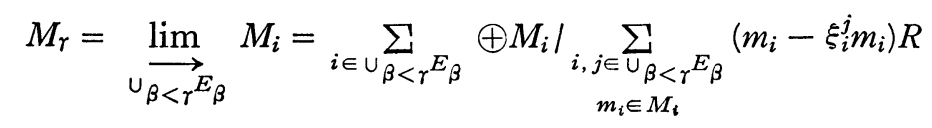

and by induction $h d_{R}\left(M_{r}\right) \leq \sup h d_{R}\left(M_{i}\right)+n$. By Proposition 0. 2,

$$
h d_{\bar{R}}\left(\sum_{i \in \cup \beta<r} E_{\beta}\left(m_{i}-\xi_{i}^{j} m_{i}\right) R\right) \leq \sup _{i \in \cup_{\beta<r} E_{\beta}} h d_{R}\left(M_{i}\right)+n-1 .
$$

By Corollary 0. 3,

$$
n+\sup h d_{R}\left(M_{i}\right) \geq h d_{R}\left(\cup_{r<\aleph_{n}} \sum_{\substack{i \in \cup_{\beta<r} E_{\beta} \\ m_{i} \in M_{i}}}\left(m_{i}-\xi_{i}^{j} r n_{i}\right) R\right) .
$$

Also, $h d_{R}\left(\Sigma \oplus M_{i}\right)=\sup h d_{R}\left(M_{i}\right)$.

But

$$
M=\underset{D}{\lim } M_{i}=\sum_{i \in D} \oplus M_{i} / \cup_{r<\aleph_{n}} \sum\left(m_{i}-\xi_{i}^{j} m_{i}\right) R
$$

so by Proposition $0.2, h d_{R}(M) \leq \sup h d_{R}\left(M_{i}\right)+n+1$.

The statement about global dimensions follows since any left ideal of $R$ is the direct limit of left ideals of the $R_{i}$ (take inverse images of the maps from the $R_{i} \longrightarrow R$ ).

Theorem 2. 3 obtains the best possible bounds, for any ideal in a valuation ring is a direct limit of projective ideals, and if it is generated by a set of $\aleph_{n}$ elements but no set of smaller cardinality, its dimention is $n+1$. And a free boolean ring on $\boldsymbol{\aleph}_{n}$ generators (global dimension $n+1$ by [13]) is a direct limit of semi-simple Artinian rings. 


\section{REFERENCES}

[1] M. Auslander: On the dimension of modules and algebras, III, Nagoya Math. J., 9 (1955), 67-77.

[2] S. Balcerzyk: On projective dimension of direct limit of modules, Bull. Acad. Polon. Sci., Sér Sci. Math. Astron. Phys. 14 (1966), 241-244.

[ 3 ] I. Berstein: On the dimension of modules and algebras, IX, Nagoya Math. J., 13 (1958), 83-84.

[4] N. Bourbaki: Algèbre commutative, Chap. 1-2, Paris, 1961.

[5] S. Eilenberg and N. Steenrod: Foundations of algebraic topology, Princeton, 1952.

[6] A.V. Jategaonkar: A counter-example in ring theory and homological algebra, Mimeographed notes, University of Rochester, 1967.

[ 7 ] C.U. Jensen: On homological dimensions of rings with countably generated ideals, Math. Scand. 18 (1966), 97-105.

[ 8 ] C.U. Jensen: Homological dimensions of $\boldsymbol{\aleph}_{0}$-coherent rings, Math. Scand. 20 (1967), $55-60$.

[ 9 ] I. Kaplansky: Projective modules, Ann. of Math. 68 (1958), 372-377.

[10] I. Kaplansky: Homological dimension of rings and modules, Mimeographed notes, University of Chicago, 1959.

[11] D. Lazard: Sur les modules plats, Comp. Rend. 258 (1964), 6313-6316.

[12] B.L. Osofsky: Global dimension of valuation rings, Trans. Amer. Math. Soc. 126 (1967), $136-149$.

[13] R.S. Pierce: The global dimension of boolean rings, to appear.

[14] J.E. Roos: Sur les foncteurs dérivés de lim. Applications., Comp. Rend. 252 (1961), 3702-3704.

Institute for Advanced Study and

Rutgers, The State University 